\title{
Fine debris flows formed by the Orientale basin
}

\author{
YuZhen Cai ${ }^{1}$, ZhiYong Xiao ${ }^{1,2,3}{ }^{*}$, ChunYu Ding ${ }^{1}$, and Jun Cui 1,3,4 \\ 1Planetary Environmental and Astrobiological Laboratory, School of Atmospheric Sciences, Sun Yat-sen University, Zhuhai, Guangdong 519000, China; \\ 2State Key Laboratory of Lunar and Planetary Sciences, Space Science Institute, Macau University of Science and Technology, Macau 999078, China; \\ ${ }^{3}$ Center for Excellence in Comparative Planetology, Chinese Academy of Science, Hefei 230026, China; \\ ${ }^{4}$ Key Laboratory of Lunar and Deep Space Exploration, National Astronomical Observatories, Chinese Academy of Sciences, Beijing 100101, China
}

\section{Key Points:}

- The radar scattering and thermophysical properties of ejecta flows of the Orientale basin are constrained

- Debris flows caused by Orientale's secondary impacts are dominated by centimeter-scale and smaller gains

- Large impact basins control the regolith structures of the entire Moon

Citation: Cai, Y. Z., Xiao, Z. Y., Ding, C. Y., and Cui, J. (2020). Fine debris flows formed by the Orientale basin. Earth Planet. Phys., 4(3), 212-222. http://doi.org/10.26464/epp2020027

\begin{abstract}
The prototype for investigations of formation mechanisms and related geological effects of large impact basins on planetary bodies has been the Orientale basin on the Moon. Its widespread secondaries, light plains, and near-rim melt flows have been well mapped in previous studies. Flow features are also widely associated with secondaries on planetary bodies, but their physical properties are not well constrained. The nature of flow features associated with large impact basins are critically important to understand the emplacement process of basin ejecta, which is one of the most fundamental processes in shaping the shallow crusts of planetary bodies. Here we use multisource remote sensing data to constrain the physical properties of flow features formed by the secondaries of the Orientale basin. The results suggest that such flows are dominated by centimeter-scale fine debris fines; larger boulders are not abundant. The shattering of target materials during the excavation of the Orientale basin, landing impact of ejecta that formed the secondaries, and grain comminution within the flows have substantially reduced particle sizes, forming the fine flows. The discovery of global-wide fine debris flows formed by large impact basins has profound implications to the interpretation of both previously-returned samples and remote sensing data.
\end{abstract}

Keywords: Moon; Orientale; impact craters; impact cratering; ejecta

\section{Introduction}

Impact basins are the most important geological units on planetary bodies. Planetary surface topography is controlled to the first order by the distribution of large impact basins (Melosh, 2011). Many basins have diameters approaching the radius of the parent body, evidence of collisions that must caused enormous thermal disturbances to the planetary interiors; e.g., the triggering of the near-global volcanism on Mercury that occurred $\sim 4.0 \mathrm{Ga}$ has been attributed to a period of massive basin formation (Marchi et al., 2013). Giant deep faults formed by the impact basins have provided weakness zones for subsequent volcanism (Spudis, 1994). Understanding the formation mechanism of impact basins and related geological effects is one of the scientific priorities in planetary exploration.

Since its initial identification, the Moon's Orientale basin has been regarded as the prototype for understanding large impact basins on planetary bodies (Hartmann, 1964). Remote sensing data have

Correspondence to: Z. Y. Xiao, xiaozhiyong@mail.sysu.edu.cn

Received 24 JAN 2020; Accepted 02 MAR 2020.

Accepted article online 16 APR 2020.

(C) 2020 by Earth and Planetary Physics. well constrained the morphological, geochemical, and geophysical properties of materials within the basin (Melosh et al., 2013). Combined with numerical simulation of impact cratering, the formation mechanism of the Orientale basin has also been well constrained (Johnson et al., 2016). The ejecta of Orientale, however, are far less well understood.

During the formation of impact basins, large amounts of materials were excavated from the transient cavity and subsequently delivered to large distances around the parent body. Therefore, impact basins and their associated ejecta deposits have long been used as global stratigraphic markers (Wilhelms, 1987). Recently, high-resolution imagery, topography, radar scattering, and reflectance spectral data have been employed to map the detailed distribution of ejecta formed by the Orientale basin (Ghent et al., 2008; Meyer et al., 2016, 2020; Guo DJ et al., 2018). Sourced from the Orientale basin, radial striations that feature high radar returns have been observed to cover most of the southern hemisphere of the lunar nearside. These deposits contain coarse gains that have been interpreted as ejecta from the Orientale basin. Secondary craters ("secondaries") formed by the Orientale basin have been mapped to a distance of $\sim 2500 \mathrm{~km}$ from the basin center (Guo DJ et al., 2018), and some may even occur globally (Meyer et 
al., 2020). The near global occurrence of light plains formed in the Orientale basin is also mapped in detail; these plains are composed by a combination of melt-bearing primary ejecta and local disturbed materials (Meyer et al., 2016). We notice that thick flow features are commonly developed around secondaries formed by the Orientale basin, and the flow features have much larger areal coverage compared to the source secondaries. By definition, the flow features are distinguishably different from the light plains, as the flows are relatively thin veneers (but still thick enough to shade topography), while light plains are smooth plain materials that were collected in local low topographic areas (Meyer et al., 2016). Similar flow features are commonly associated with all secondaries on planetary bodies, but those formed by the Orientale basin have not previously been discussed.

Considering that flow features can cover surfaces at least as large as the source secondaries, resolving their physical properties is necessary to any comprehensive understanding of the formation of the Orientale basin and also of the surface processes on the Moon. In this study, we use multi-source high-resolution remote sensing data to constrain the physical properties of the flows. Ways in which flow features can elucidate planetary surface evolution are discussed.

\section{Data and Method}

Images obtained with large incidence angles by the wide-angle camera of the Lunar Reconnaissance Orbit Camera (LROC WAC; $100 \mathrm{~m} /$ pixel; Robinson et al., 2010) and the global mosaic obtained by the Chang'e- 2 mission $(50 \mathrm{~m} /$ pixel; Zhao $\mathrm{HX}$ and Magoulès, 2011) are used to study the morphology of ejecta deposits from the Orientale basin. Flow features formed by Orientale's secondaries are selected as the research target.

Topography of flow features is derived using the Selene-LOLA-Digital Elevation model (SLDEM) with $59.2 \mathrm{~m} /$ pixel spatial resolution (Barker et al., 2016). Thicknesses of flows are measured based on topographic profiles that are perpendicular to the flows. The measured values are lower estimates, considering that the flows have been degraded after $\sim 3.8$ billion years of exposure to gravity and subsequent impact (Spudis, 1994).

Thermophysical properties of the flows are studied using data obtained by the Lunar Reconnaissance Orbiter Diviner thermal radiometer (Paige et al., 2010). Seven of the radiometer's nine channels are in the infrared, covering $\sim 800 \mu \mathrm{m}$ in wavelength (Paige et al., 2010). Two global gridded products used in this study, rock abundance (Bandfield et al., 2011) and $\mathrm{H}$-parameters (Hayne et al., 2017), are derived from the night-time temperature data obtained by Diviner. These two parameters are calculated based on the observation that materials with higher thermal inertia (e.g., competent rocks) sustain temperatures higher during the lunar night than do the lower-thermal-inertia fine regolith materials. The quasi-physical meaning of the rock abundance values is the areal-fraction of each field of view that is occupied by surface high-thermal-inertia materials. A rock abundance value of 0.01 means that $1 \%$ of the relevant pixel is occupied by exposed highthermal-inertia materials (e.g., competent boulders) that have sizes larger than the diurnal thermal skin depth $(\sim 1 \mathrm{~m}$ on the Moon; Bandfield et al., 2011). The rock abundance map covers all longitudes and latitudes from $80^{\circ} \mathrm{S}$ to $80^{\circ} \mathrm{N}$, and spatial resolution is 128 pixels per degree ( $236 \mathrm{~m} /$ pixel at the equator). The rock abundance data are available at http://pds-geosciences.wustl.edu/ missions/Iro/diviner.htm. $H$-value is another expression of the thermal inertia of lunar surface materials (Hayne et al., 2017); the Moon's global average value of the $H$-parameter is $\sim 0.68$. Larger $\mathrm{H}$-parameter values indicate more insulating material near the surface, and smaller values suggest denser, more conductive material. The gridded map of $H$-values (128 pixel/degrees) covers all longitudes, but its latitude coverage is limited to $60^{\circ} \mathrm{S}$ to $60^{\circ} \mathrm{N}$. It has been noticed that, for some purposes, $H$-values are more sensitive than rock abundance data to the actual thermal inertia of surface materials. H-value map data are accessible from https:// phayne.github.io/research/.

Radar observations of flow features formed by the Orientale basin are performed using P-band ( $f=430 \mathrm{MHz}$, or $70 \mathrm{~cm}$ wavelength) and S-band ( $f=2380 \mathrm{MHz}$, or $12.6 \mathrm{~cm}$ wavelength) data obtained by both the LRO Miniature Radio Frequency (Mini-RF; Nozette et al. 2010) and Earth-based radar images acquired in bistatic geometry from the Arecibo and Green Bank telescopes (Campbell et al., 2007, 2010). The radar data at different wavelengths are used together to estimate semi-quantitatively the sizes of particles at different flow depths (Ghent et al., 2016). A rule of thumb for radar detection on the Moon is that radar waves are sensitive to scatters that are at least 1/10th of the radar wavelengths, and that the radar waves can penetrate in lunar materials down to a depth of 10 times the radar wavelengths (Ghent et al., 2016). The global gridded map of the Mini-RF data that were collected at S-band wavelength $(12.6 \mathrm{~cm})$ are used in this study. The spatial resolution is 128 pixels/degree. The spatial resolution of the $12.6 \mathrm{~cm}$ wavelength radar data obtained from Earth telescopes is $\sim 80$ $\mathrm{m} /$ pixel; that of the $70 \mathrm{~cm}$ wavelength radar data is $\sim 400 \mathrm{~m} /$ pixel. Avoiding difficult observation geometries due to unfavorable topography and satellite positions, we use the circular polarization ratio (i.e., $\mathrm{CPR}$ ) as the parameter to investigate the bulk roughness of the flow feature; this technique yields a good estimate of the abundance of scatters within the flows. The global average value of CPR values is 0.4 (Campbell et al., 2006, Carter et al., 2009).

Note that the above datasets have different spatial coverage and resolutions. Large offsets exist in the radar data compared to the well-controlled imagery and topography data. Therefore, while selecting flow features that were formed by the Orientale basin, we have done careful de-referencing for the different datasets, and have chosen only obvious flows that have widths of at least $1 \mathrm{~km}$ and for which the available data have good signal-to-noise ratios.

\section{Results}

We have selected 9 locations of Orientale secondaries on the lunar nearside that have data coverage by Earth-based P-band and Sband radar (Campbell et al., 2007, 2010). Figure 1 shows the locations of the selected examples, which are $\sim 800-1500 \mathrm{~km}$ from the center of Orientale. At regional scales, the selected examples contain subareas that have CPR both larger than and comparable to the global average. Beyond the final topographic rim of the Orientale basin, an annular region that has lower CPR values is visible in both the $70 \mathrm{~cm}$ and the $12.6 \mathrm{~cm}$ wavelengths radar data. 

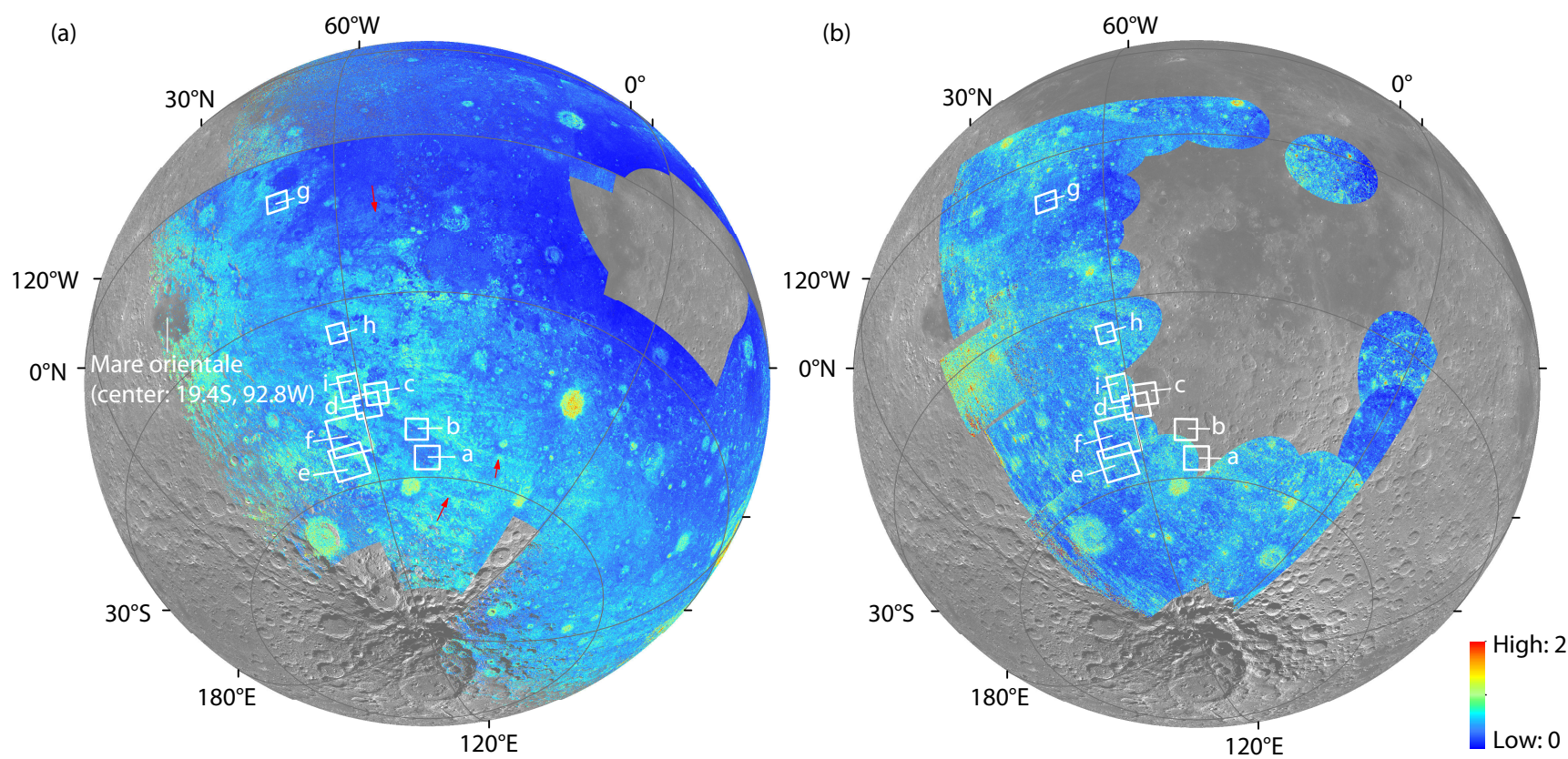

Figure 1. Earth-based $70 \mathrm{~cm}$ (a) and $12.6 \mathrm{~cm}$ (b) wavelengths of radar data showing the ejecta deposits of Orientale at the southern hemisphere of the lunar nearside. The base image is the global mosaic obtained by the Chang'e-2 mission. Red arrows denote the radial striations from Orientale that exhibit a higher CPR. The white boxes outline the interested areas that have been studied in detail and are mapped in Figure 2.

\subsection{Morphology and Topography of the Flows Formed by Orientale's Secondaries}

Flow features are commonly associated with secondaries formed by the Orientale basin. The flows have rather continuous extension along the chains or clusters of secondaries, and clear flow lines are visible along the path (Figure 2). The flows can flow across local topographies; deposition along the path is not substantial since the surface elevations along the flows are not obviously changed if other possible deposition has occurred. Flow margins are most obvious in directions perpendicular to the flow paths (Figure 2).

The minimum thickness of the flows is estimated along directions perpendicular to the flow paths. During this process, complicated background topography, where the flows have move across, has been avoided. The topographic profiles shown in Figure 2 reveal that the flows have different thicknesses, varying from 120-420 m.

\subsection{Thermophysical Properties of the Flow Features}

The ejecta deposits of the Orientale basin do not exhibit abnormal radial patterns around the crater (Bandfield et al., 2011). Figure 3 shows rock abundance and $\mathrm{H}$-parameter maps for the same region shown in Figure 1. The radial dark and bright striations that are visible in the radar data are no longer obvious in either the $\mathrm{H}$-parameter or the rock abundance map. Instead, fresh impact rays, and walls and blocky rims of impact craters are the most obvious features in the global thermophysical maps (Bandfield et al., 2011). This is consistent with the fact that regolith production on the Moon was fast enough that lunar surfaces older than $\sim 1$ billion years have been covered quickly by a regolith layer that is thick enough to homogenize the thermophysical properties (Bandfield et al., 2011). Therefore, as seen in the Diviner data, the flow features formed by the Orientale's secondaries are not expected to exhibit abnormally low or high thermal inertia.

\subsection{CPR Values for the Flow Features Derived from Earth- Based S-Band Radar Data}

Earth-based S-band radar data are used to investigate the flow features shown in Figure 4. Mini-RF S-band data can also be used, but Earth-based radar data have larger image frames so that georeferencing is not as laborious, compared to use of Mini-RF data (Fa WZ and Eke, 2018).

The $S$ band CPR values for the flow features show that regions exist with CPR values both larger and smaller than 0.4. Close inspection reveals that the majority of the smooth parts of the flow features exhibit CPR values less or comparable to 0.4 (Figures 2 and 4). Higher CPR regions are mostly correlated with walls of impact craters and with topographic ridges in the floor, where coarser materials are more prone to be exposed due to continuous mass wasting (Xiao ZY et al., 2013).

\subsection{CPR Values for the Flow Features Derived from the P- Band Radar Data}

The flows are immediately apparent on the P-band CPR maps (Figure 5). Compared to the features shown in the S-band CPR maps, the flows are more homogeneous in terms of CPR values in the P-band maps. The majority of flow features have CPR values less than 0.4 , suggesting that materials within the flows have sizes comparable with those of typical lunar regolith. In the P-band CPR maps, only rims of relative large craters exhibit high CPR values.

\section{Discussion}

\subsection{Physical Properties of Orientale's Distant Ejecta Deposits}

Integrating the morphological, geometric, thermophysical, and radar properties of the flow features suggests that the flows are 

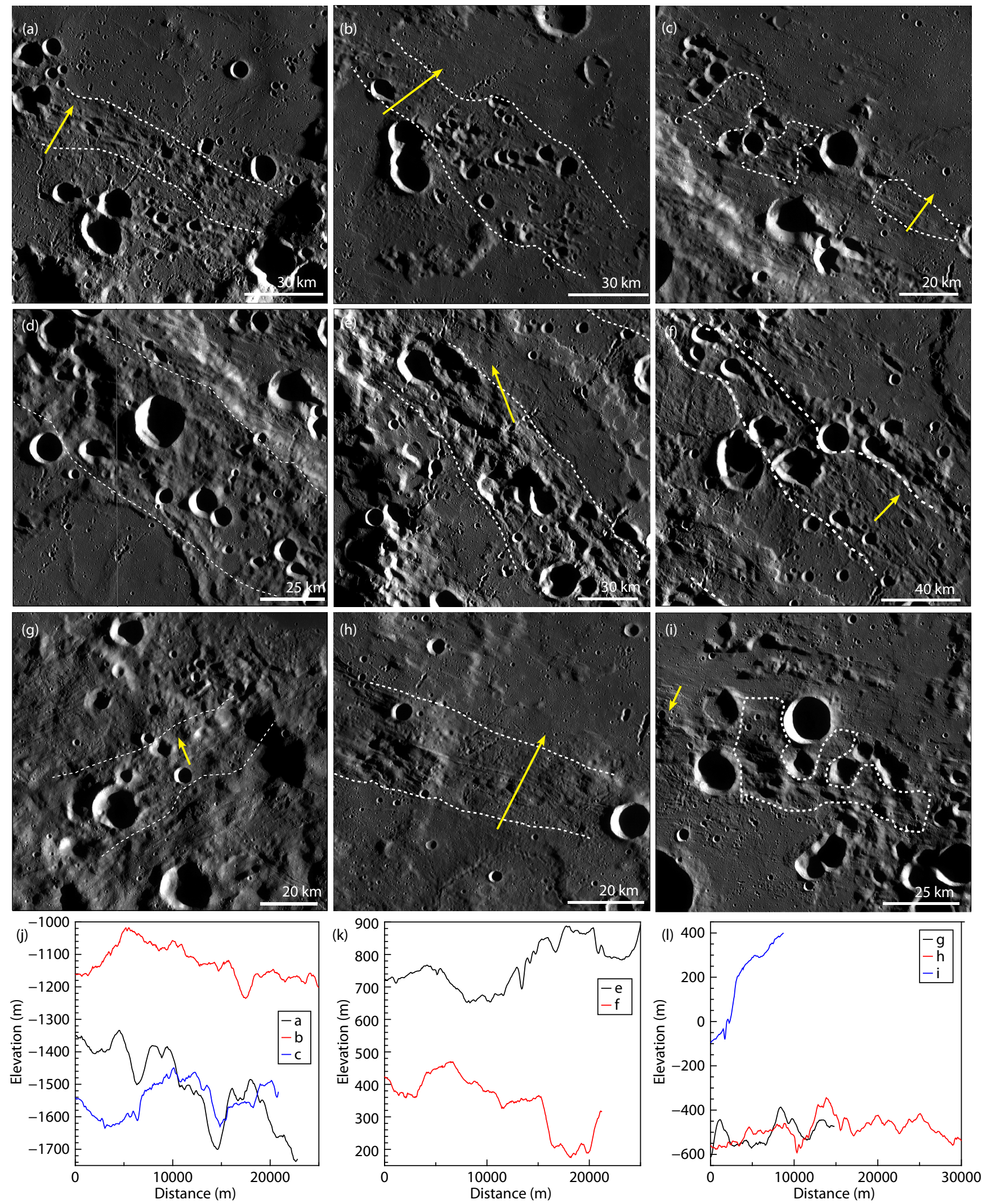

Figure 2. Morphology and topography of flow features associated with Orientale's secondaries. The locations of the flow features are marked in Figure 1. North is up in all the images. Panels (a)-(i) are LROC WAC mosaics showing the morphology of the flows. White dashed lines denote the approximate flow boundaries seen in the $100 \mathrm{~m} /$ pixel mosaics. Yellow dashed lines are locations along which topographic data were extracted based on SLDEM. The right-most panels show the topographic profiles derived for each of the flows shown in the same row. The minimum flow thicknesses are derived along the profile by comparing with the monochrome mosaics. 

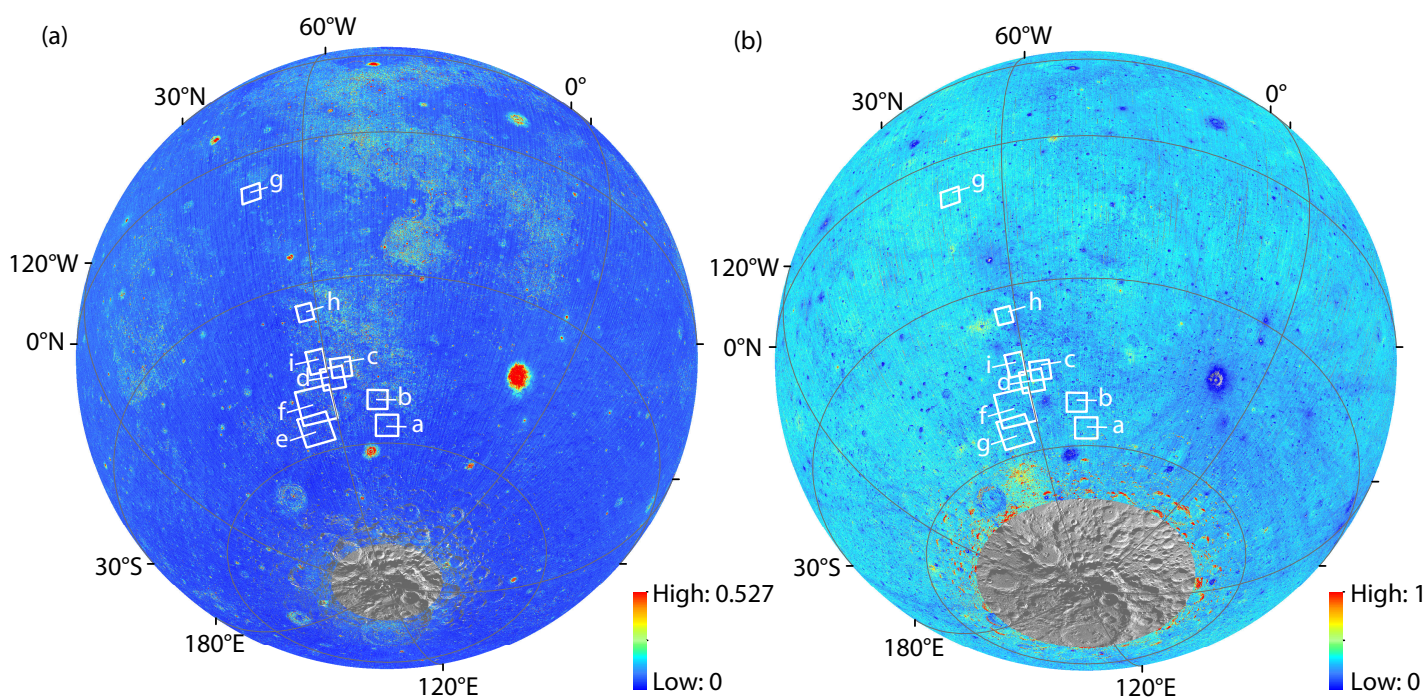

Figure 3. Thermophysical properties of ejecta deposits around the Orientale basin. Unlike the radar data, in which the ejecta deposits formed by the Orientale basin preserve the radial distribution pattern in terms of CPR values (Figure 1), both the rock abundance (a) and $H$-parameters (b) show no unambiguous radial deposits from the Orientale basin. Both panels use as basemaps the global mosaic obtained by Chang'e-2.
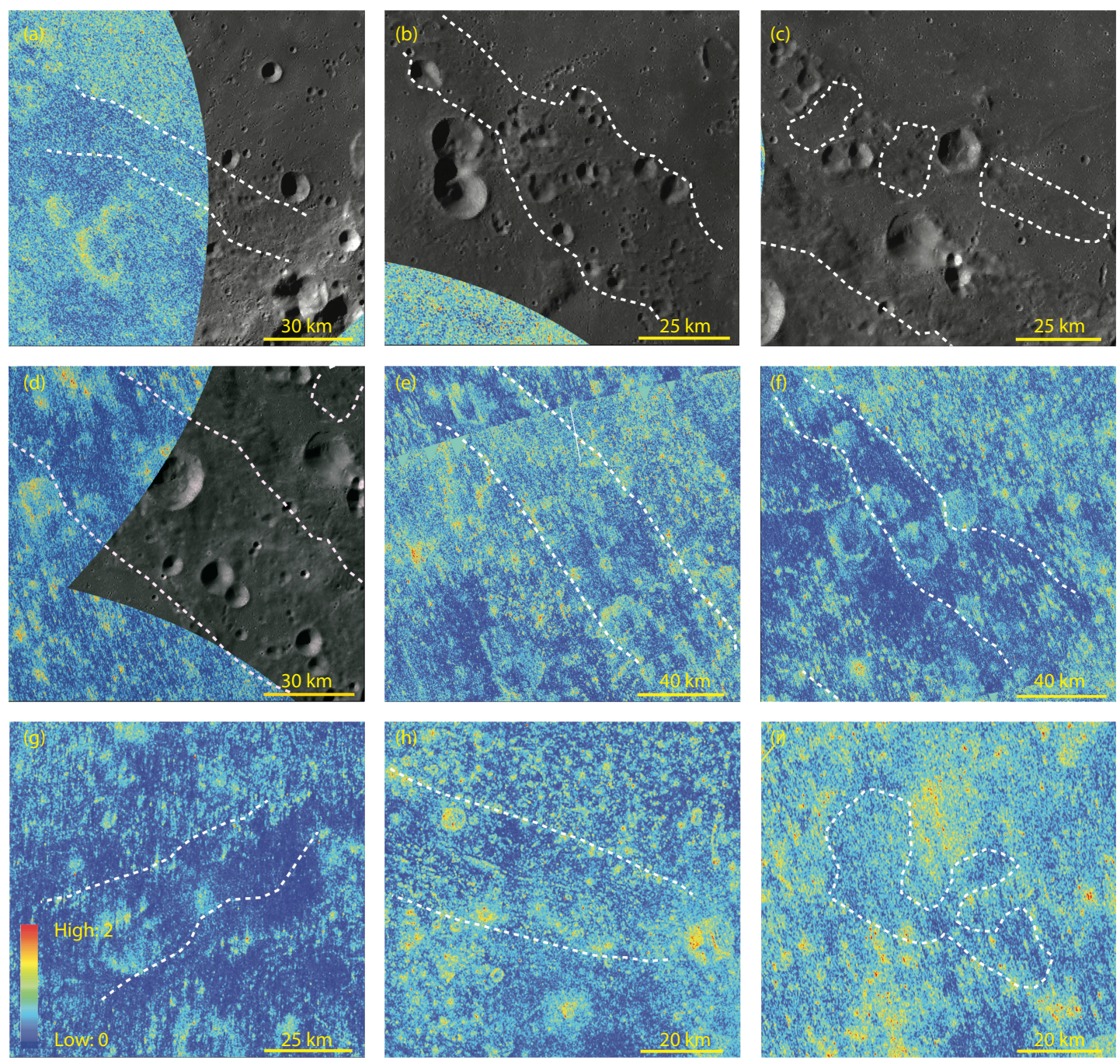

Figure 4. CPR values of S-band radar data obtained by Earth based radars. The CPR values are restricted to the range of 0-2; the deep blue color corresponds to CPR values less than 0.4. Most of the smooth parts of the flows exhibit CPR values less or comparable to 0.4. Note that portions of the flows shown in panels (b)-(d) are not covered by the S-band radar data. 

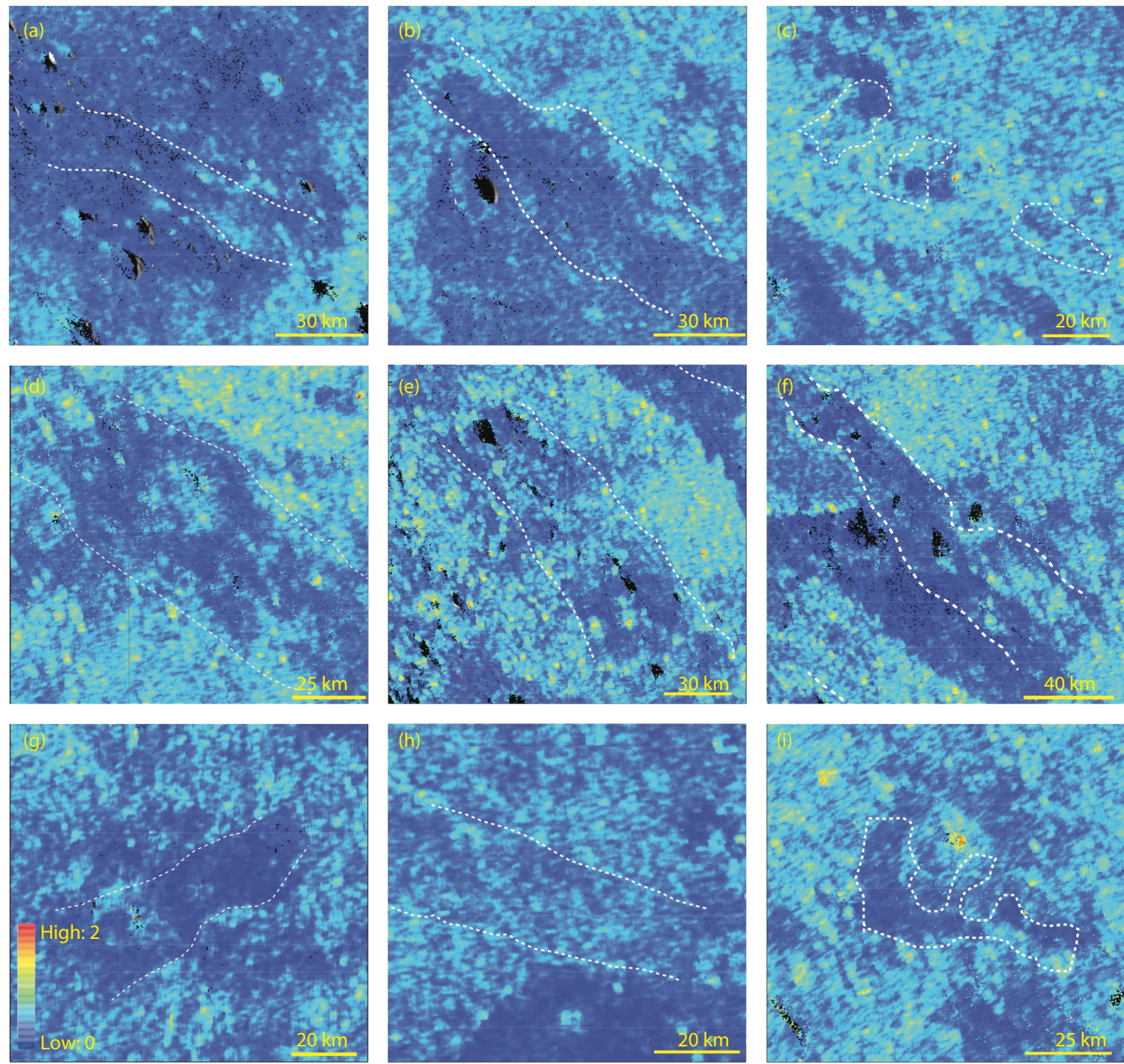

Figure 5. CPR maps derived from P-band Earth-based radar data for the 9 flows associated with Orientale's secondaries (Figure 2). The flows exhibit more homogeneous CPR values, and most the flow materials have CPR values less than 0.4.

composed primarily of grains less than $\sim 7 \mathrm{~cm}$ in dimension (Table 1). While the average rock abundance and $\mathrm{H}$-values are comparable to the global average $\left(H_{\text {average }}=0.68\right.$; Hayne et al., 2017), the flows uniformly exhibit CPR values less than 0.4 at the P-band radar. The average CPR values for the S-band radar data can be as large as $\sim 0.72$ (Table 2). Controlled comparison between the CPR map and surface morphology suggests that the $\mathrm{S}$-band CPR values are strongly affected by the fractions of small topographies, such as crater walls and scarps. The content of impact melt in the flows should be low considering the low CPR values.

For the continuous ejecta deposits of Orientale, our observations here are basically consistent with P-band radar observations. This region occurs as a radar dark halo around the Orientale basin (Ghent et al., 2005), and flow features have entirely covered this region. Based on the radar properties, Ghent et al. (2005) suggested that the Orientale basin is similar to many complex craters on the Moon, that a low-radar-return haloed region exists beyond the blocky ejecta deposits. We suggest that these materials are at least $7 \mathrm{~m}$ thick, and dominated by grains less than $7 \mathrm{~cm}$ in dimensions.

\subsection{Formation Mechanism of the Fine Debris Flows}

The flows observed here are spatially associated with secondaries. Secondaries of Orientale are as large as $\sim 20 \mathrm{~km}$ in diameter (Xiao ZY and Werner, 2015). While the primary ejecta that formed the secondaries have contributed a substantial portion of materials in the flows (Schultz and Gault, 1985; Hawke et al., 2003), locally excavated materials are entrained in the excavated materials by the secondaries. Similar observations of secondaries-related flow features are widely observed on both the Moon (Lucchitta, 1972), Mercury (Xiao ZY, 2016), and Mars (Ghent et al., 2010).

During impact cratering, excavated materials are strongly shattered due to shock, and post-launch fragmentation occurrs if the residual elastic strain in the ejecta is large enough to overcome the tensile strength (Melosh, 1984, 1989). Upon impact, shock or elastic loading occurs depending on the landing velocity, so that the primary ejecta are further shattered during the forma- 
Table 1. Locations, minimum thicknesses, average CPR values at P-and S-band radar, and average rock abundance and $H$-parameters for the 9 flow features shown in Figure 2.

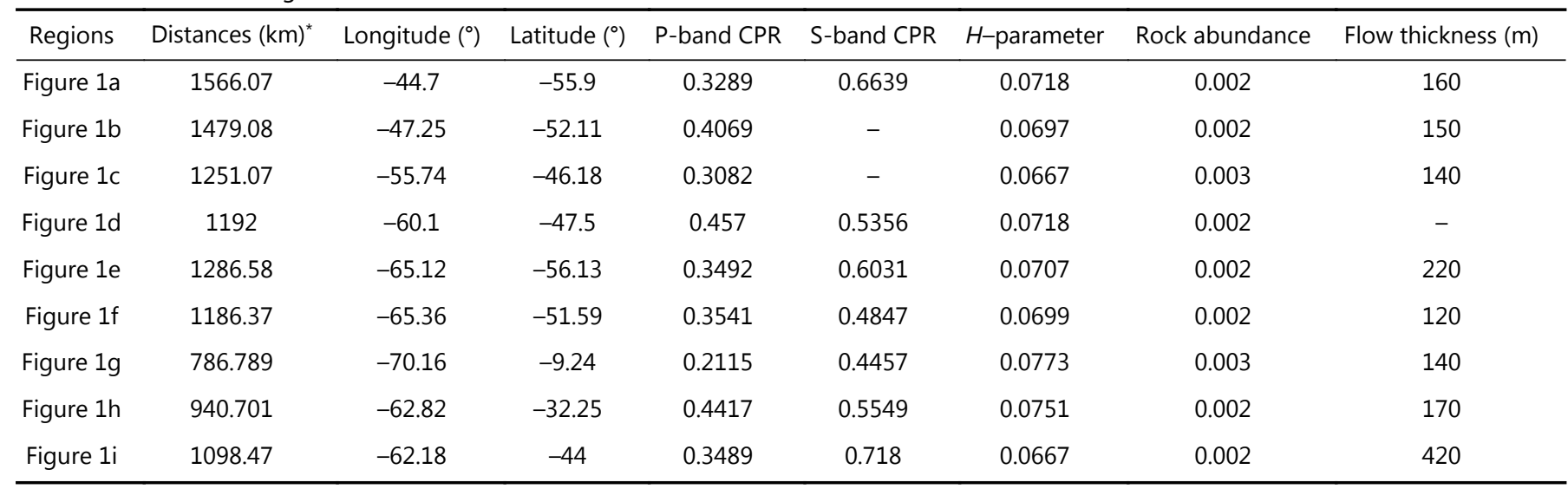

* The distances are measured along great circles to the center of Orientale.

Table 2. Locations, minimum thicknesses, and average CPR values at S-band radar for the 9 flow features shown in Figure 2.

\begin{tabular}{cccccc}
\hline Regions & Distance $(\mathrm{km})^{*}$ & Longitude $\left(^{\circ}\right)$ & Latitude $\left(^{\circ}\right)$ & Mini-RF S-band CPR & Minimum flow thickness $(\mathrm{m})$ \\
\hline Figure 6b & 1969.09 & -138.237 & 29.695 & 0.5605 & 200 \\
Figure 6c & 1797.42 & -139.91 & 19.49 & 0.5358 & 260 \\
Figure 6d & 1107.05 & -128.52 & -4.18 & 0.5118 & 270 \\
Figure 6e & 933.872 & -127.02 & -29.42 & 0.5259 & 150 \\
Figure 6f & 1644.89 & -153.31 & -46.06 & 0.5148 & 130 \\
Figure 6g & 2631.12 & -167.62 & 31.66 & 0.5321 & 80 \\
\hline
\end{tabular}

* The distances are measured along great circles referred to the center of Orientale.

tion of secondaries. However, many impact rays on the Moon and Mercury exhibit CPR values larger than 0.4 (Neish et al., 2013), so that the ejecta deposits are not small enough upon landing. Further fragmentation occurs when the flows are initiated by the secondaries. Grain collisions consume the kinetic energy of the flows, promoting further fragmentation.

\subsection{Widespread Farside Debris Flows Associated with Orientale}

Similar flow features associated with Orientale's secondaries are also commonly observed at the lunar farside. Figure 6 shows examples that have radial distances of $\sim 900-2000 \mathrm{~km}$ from the center of the Orientale basin. The flows feature morphology similar to those shown in Figure 2 and the minimum flow thicknesses are $\sim 80-270 \mathrm{~m}$.

Similar to the CPR values at S-band for the nearside flow features, the flows at the lunar farside also exhibit a large range of CPR values in the Mini-RF S-band data, but the smooth parts exhibit CPR values lower or comparable to 0.4 (Figure 7, Table 2). This observation further suggests that fine-grained flows formed by Orientale's secondaries at both the nearside and farside share similar physical properties.

\subsection{Relationship Between Flows Formed by Secondaries and Light Plains}

Flow features could be sources of at least some of the material forming the light plains on Orientale (Figure 6). Light plains are composed of impact breccia, similar to the Cayley Plains visited by the Apollo 16 mission, which were formed by the Imbrium basin. Light plains do not have characteristic reflectance spectra in visible-to-near infrared wavelengths. The general content of melt within the plains is much lower than that of typical low-reflectance melt ponds (Meyer et al., 2020). The similar distribution ranges of secondaries and light plains suggest that many secondaries-initiated flows are partly connected with the light plains (Figure 6), contributing to material deposition at topographic lows.

\subsection{Challenge to Current Ballistic Sedimentation Models}

Blanking and mixing of local materials by secondary impacts have long been taken as the most important mechanisms in exchange materials across the Moon (Petro and Pieters, 2006). It is well accepted now that ballistic sedimentation is not via simple blanketing, since excavation of local material can be a dominant component in deposits of secondaries (Oberbeck, 1975). However, considering excavation by secondaries, the largest depth of homogenneous mixing is equal to or less than the thickness of the primary ejecta (Petro and Pieters, 2006).

A comparison between predictions of primary ejecta thicknesses based on prevalently-used empirical functions (Pike, 1974; Xie MG and Zhu MH, 2016) and the observed minimum thicknesses of ejecta flows suggests that the empirical functions have substan- 

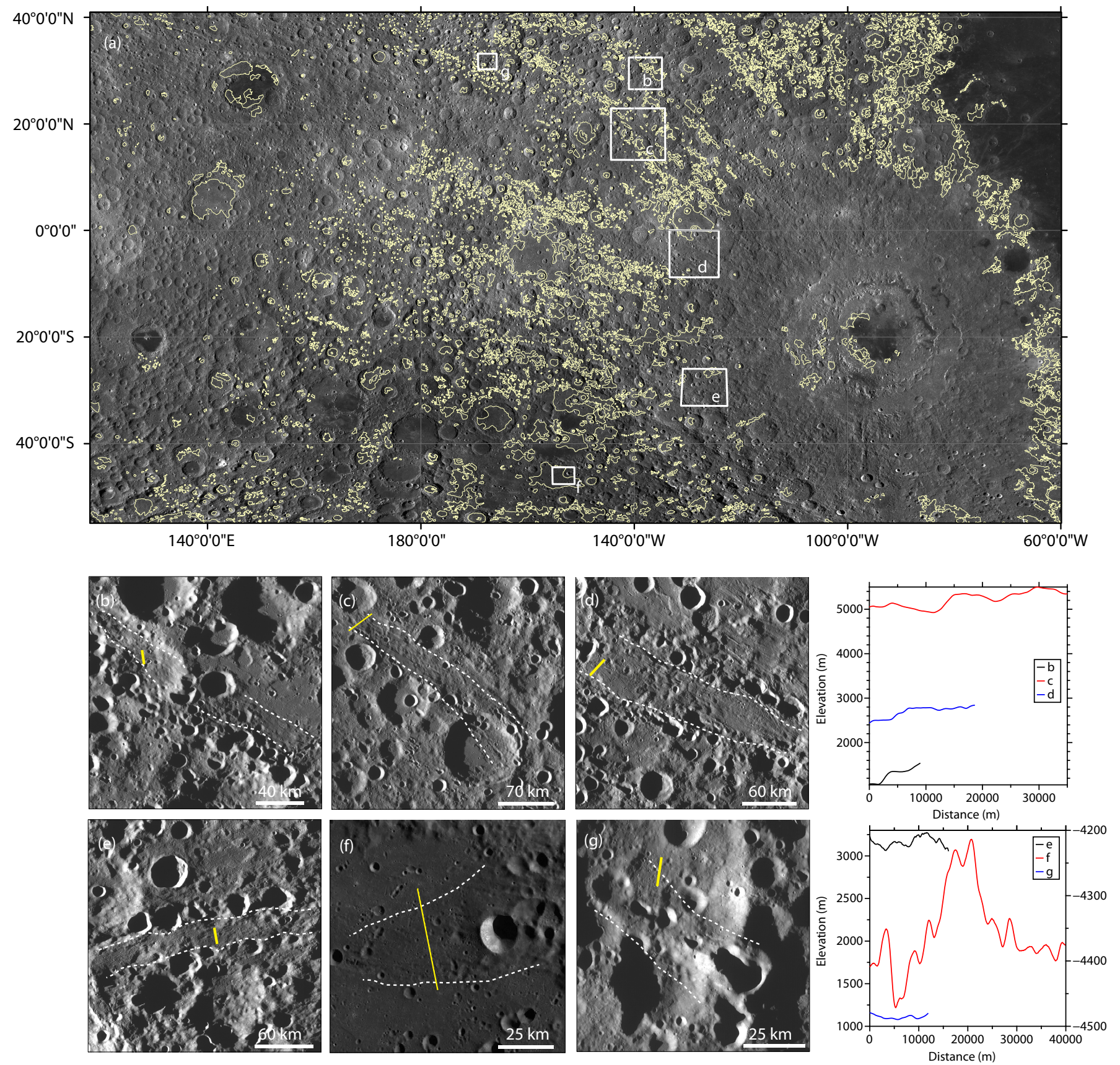

Figure 6. Morphology and topography of flows formed by Orientale's secondaries at the lunar farside. (a) The distribution of Orientale's lightplains, mapped secondaries, and the exemplified flow features that are associated with Orientale's secondaries at the lunar farside. The distribution of light plains is referred from Meyer et al. (2016), and the distribution of interpreted secondaries of Orientale is denoted as green dots. The base mosaic is obtained by Chang'e-2. The white boxes mark locations of the flow features shown in panels (b)-(g), which are based on the LROC WAC global mosaic. Topographic profiles for thickness estimates for the flow features are shown in the right panels. In panels (b)-(g), the locations of the profiles are denoted as white dashed lines.

tially underestimated the thickness of ejecta deposits of secondaries. Figure 8 shows the comparison. Flow thicknesses can be as large as $\sim 15$ times the predicted thickness of primary ejecta, suggesting that the flows are dominated by locally excavated materials; currently available ballistic sedimentation models need to consider the widespread flow features associated with secondary impacts.

This discovery brings intriguing questions about the provenances of returned samples. The Apollo samples were believed to be largely contaminated by the primary ejecta from the Imbrium basin (Spudis et al., 2011). However, flows formed by Imbrium's secondaries should be abundant across the Moon; such flows are dominated by materials excavated by the secondaries, and minor impact melt materials formed by Imbrium should exist in the flows. A re-interpretation of sample provenances and their indications regarding the Moon's early impact history (Hartmann, 2019) will be needed. 

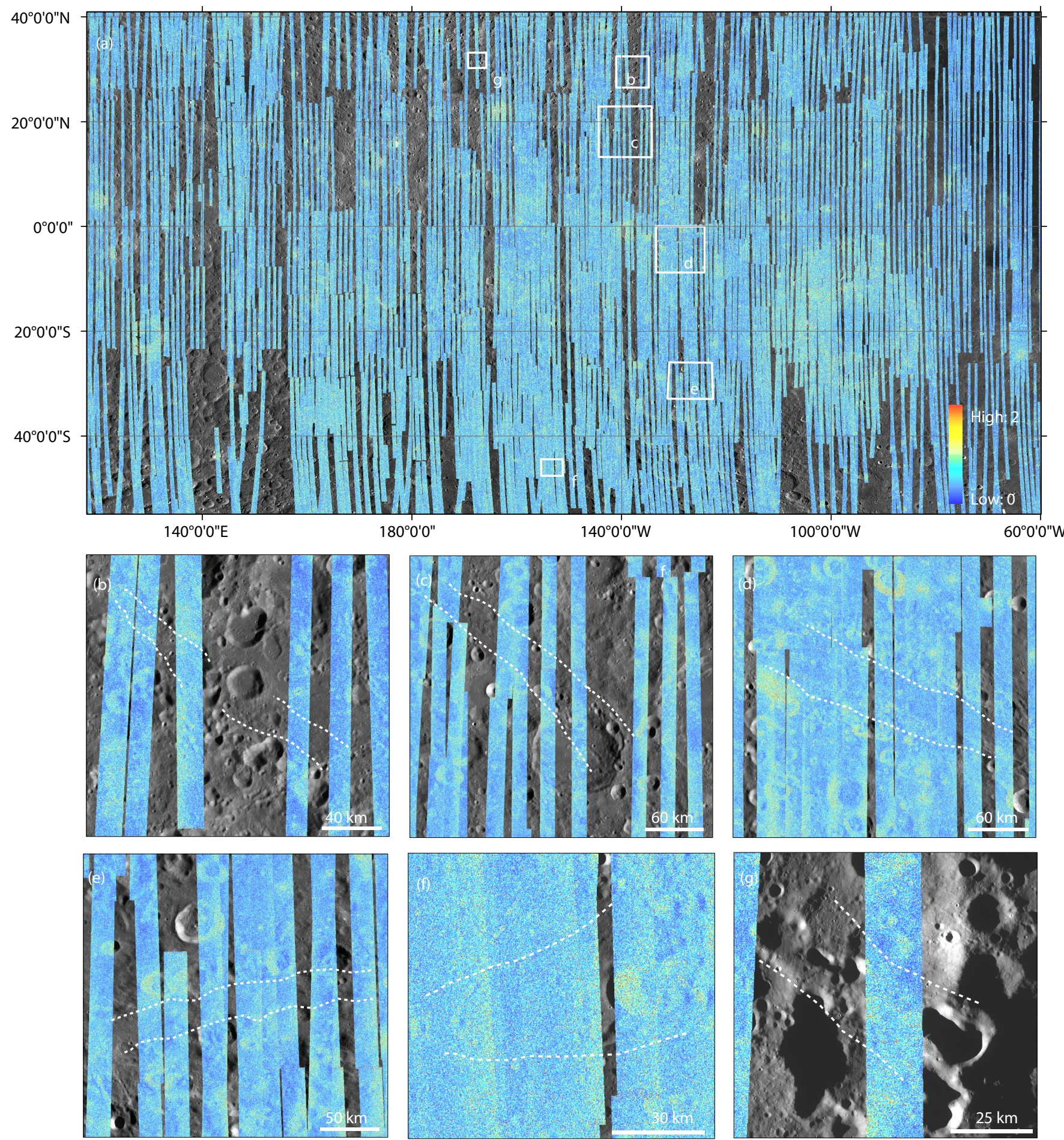

Figure 7. Flows formed by Orientale's secondaries at the lunar farside feature CPR values similar to those of the nearside flows. (a) CPR map for the lunar farside revealed by the S-band Mini-RF data. (b)-(g) show regional CPR maps for the 6 flows in Figure 6. A close comparison between morphology and CPR values suggests that the smooth parts of the flows exhibit CPR values of about 0.4.

\section{Conclusion}

We have found that widespread flow features are associated with the secondaries of the Orientale basin. The thermophysical and radar properties of the flows are compared in detail, together with morphology and topography data. Our results suggest that the flows are dominated by centimeter-scale grains, which were formed during the excavation stage of the Orientale basin, the landing of the ejecta, and subsequent movements of the flows. Our analysis suggests that secondaries-related flows were an important agent in shaping the lunar shallow crust, and that analyses of returned samples and design of future missions should consider the wide occurrence and importance of secondaries-related flows. 


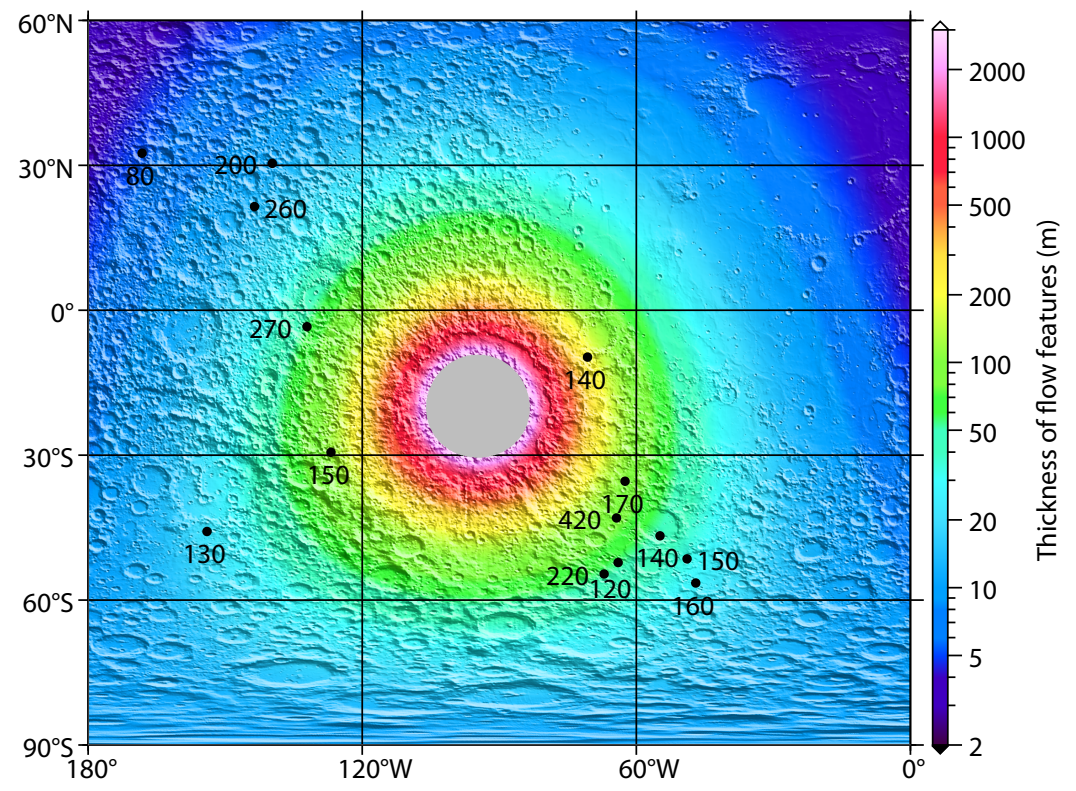

Figure 8. A comparison between the predicted ejecta thickness at various radial distances from the center of the Orientale basin using the empirical function of Pike (1974) and the measured minimum thickness of the flow features shown in Figures 2 and 5 . Note that other empirical models are available, but the results are comparable within an order of magnitude. Our observations here show a strong mismatch between observation and prediction, especially at areas $>1500 \mathrm{~km}$ from the basin center. In this plot, the transient cavity of the Orientale basin is referred from Melosh et al. (2013); the rim-to-rim radius of the transient crater is $234 \mathrm{~km}$.

\section{Acknowledgments}

All data used in this study are in the public domain. This work is supported by the B-type Strategic Priority Program of the Chinese Academy of Sciences, Grant No. XDB41000000, the National Natural Science Foundation of China (41773063, 41525015 and 41830214), the Science and Technology Development Fund of Macau (0042/2018/A2), and the Pre-research Project on Civil Aerospace Technologies (No. D020101) of CNSA.

\section{References}

Bandfield, J. L., Ghent, R. R., Vasavada, A. R., Paige, D. A., Lawrence, S. J., and Robinson, M. S. (2011). Lunar surface rock abundance and regolith fines temperatures derived from LRO Diviner radiometer data. J. Geophys. Res.: Planets, 116(E12), E00H02. https://doi.org/10.1029/2011JE003866

Barker, M. K., Mazarico, E., Neumann, G. A., Zuber, M. T., Haruyama, J., and Smith, D. E. (2016). A new lunar digital elevation model from the Lunar Orbiter Laser Altimeter and SELENE Terrain Camera. Icarus, 273, 346-355. https://doi.org/10.1016/j.icarus.2015.07.039

Campbell, B. A., Campbell, D. B., Margot, J. L., Ghent, R. R., Nolan, M., Chandler, J., Carter, L. M., and Stacy, N. J. S. (2007). Focused 70-cm wavelength radar mapping of the Moon. IEEE Trans. Geosci. Remote Sens., 45(12), 4032-4042. https://doi.org/10.1109/TGRS.2007.906582

Campbell, B. A., Carter, L. M., Campbell, D. B., Nolan, M., Chandler, J., Ghent, R. R., Hawke, B. R., Anderson, R. F., and Wells, K. (2010). Earth-based 12.6-cm wavelength radar mapping of the Moon: New views of impact melt distribution and mare physical properties. Icarus, 208(2), 565-573. https://doi.org/10.1016/j.icarus.2010.03.011

Campbell, D. B., Campbell, B. A., Carter, L. M., Margot, J. L., and Stacy, N. J. S. (2006). No evidence for thick deposits of ice at the lunar south pole. Nature, 443(7113), 835-837. https://doi.org/10.1038/nature05167

Carter, L. M., Campbell, B. A., Hawke, B. R., Campbell, D. B., and Nolan, M. C. (2009). Radar remote sensing of pyroclastic deposits in the southern Mare Serenitatis and Mare Vaporum regions of the Moon. J. Geophys. Res: Planets, 114(E11), E11004. https://doi.org/10.1029/2009JE003406
Fa, W. Z., and Eke, V. R. (2018). Unravelling the mystery of lunar anomalous craters using radar and infrared observations. J. Geophys. Res.: Planets, 123(8), 2119-2137. https://doi.org/10.1029/2018JE005668

Ghent, R. R., Leverington, D. W., Campbell, B. A., Hawke, B. R., and Campbell, D. B. (2005). Earth-based observations of radar-dark crater haloes on the Moon: Implications for regolith properties. J. Geophys. Res.: Planets, 110(E2), E02005. https://doi.org/10.1029/2004JE002366

Ghent, R. R., Campbell, B. A., Hawke, B. R., and Campbell, D. B. (2008). Earthbased radar data reveal extended deposits of the Moon's Orientale basin. Geology, 36(5), 343-346. https://doi.org/10.1130/G24325A.1

Ghent, R. R., Gupta, V., Campbell, B. A., Ferguson, S. A., Brown, J. C. W., Fergason, R. L., and Carter, L. M. (2010). Generation and emplacement of fine-grained ejecta in planetary impacts. Icarus, 209(2), 818-835. https://doi.org/10.1016/j.icarus.2010.05.005

Ghent, R. R., Carter, L. M., Bandfield, J. L., Udovicic, C. J. T., and Campbell, B. A. (2016). Lunar crater ejecta: Physical properties revealed by radar and thermal infrared observations. Icarus, 273, 182-195. https://doi.org/10.1016/j.icarus.2015.12.014

Guo, D. J., Liu, J. Z., Head III, J. W., and Kreslavsky, M. A. (2018). Lunar orientale impact basin secondary craters: Spatial distribution, size-frequency distribution, and estimation of fragment size. J. Geophys. Res.: Planets, 123(6), 1344-1367. https://doi.org/10.1029/2017JE005446

Hartmann, W. K. (1964). Radial structures surrounding lunar basins, II: Orientale and other systems; Conclusions. Commun. Lunar Planet. Lab., 2(36), 175-192.

Hartmann, W. K. (2019). History of the terminal cataclysm paradigm: Epistemology of a planetary bombardment that never (?) happened. Geosciences, 9(7), 285. https://doi.org/10.3390/geosciences9070285

Hawke, B. R., Peterson, C. A., Blewett, D. T., Bussey, D. B. J., Lucey, P. G., Taylor, G. J., and Spudis, P. D. (2003). Distribution and modes of occurrence of lunar anorthosite. J. Geophys. Res.: Planets, 108(E6), 5050. https://doi.org/10.1029/2002JE001890

Hayne, P. O., Bandfield, J. L., Siegler, M. A., Vasavada, A. R., Ghent, R. R., Williams, J. P., Greenhagen, B. T., Aharonson, O., Elder, C. M., .. Paige, D. A. (2017). Global regolith thermophysical properties of the moon from the Diviner lunar radiometer experiment. J. Geophys. Res.: Planets, 122(12), 2371-2400. https://doi.org/10.1002/2017JE005387

Johnson, B. C., Blair, D. M., Collins, G. S., Melosh, H. J., Freed, A. M., Taylor, G. F., 
Head, J. W., Wieczorek, M. A., Andrews-Hanna, A. C., ... Zuber, M. T. (2016). Formation of the Orientale lunar multiring basin. Science, 354(6311), 441-444. https://doi.org/10.1126/science.aag0518

Lucchitta, B. K. (1972). The Apollo 17 landing site. Nature, 240(5379), 259-260. https://doi.org/10.1038/240259a0

Marchi, S., Chapman, C. R., Fassett, C. I., Head, J. W., Bottke, W. F., and Strom, R. G. (2013). Global resurfacing of Mercury 4.0-4.1 billion years ago by heavy bombardment and volcanism. Nature, 499(7456), 59-61. https://doi.org/10.1038/nature12280

Melosh, H. J. (1984). Impact ejection, spallation, and the origin of meteorites. Icarus, 59(2), 234-260. https://doi.org/10.1016/0019-1035(84)90026-5

Melosh, H. J. (1989). Ejecta deposits. In H. Charnock et al. Impact Cratering: A Geologic Process (pp. 87-110). New York: Oxford Univ. Press.

Melosh, H. J. (2011). Impact cratering. In H. J. Melosh (Ed.), Planetary Surface Processes (pp. 222-275). Cambridge: Cambridge University Press. https://doi.org/10.1017/CBO9780511977848

Melosh, H. J., Freed, A. M., Johnson, B. C., Blair, D. M., Andrews-Hanna, J. C., Neumann, G. A., Phillips, R. J., Smith, D. E., Solomon, S. C., ... Zuber, M. T. (2013). The origin of lunar mascon basins. Science, 340(6140), 1552-1555. https://doi.org/10.1126/science.1235768

Meyer, H. M., Denevi, B. W., Boyd, A. K., and Robinson, M. S. (2016). The distribution and origin of lunar light plains around Orientale basin. Icarus, 273, 135-145. https://doi.org/10.1016/j.icarus.2016.02.014

Meyer, H. M., Denevi, B. W., Robinson, M. S., and Boyd, A. K. (2020). The global distribution of lunar light plains from the lunar reconnaissance orbiter camera. J. Geophys. Res.: Planets, 125(1), e2019JE006073. https://doi.org/10.1029/2019JE006073

Neish, C. D., Blewett, D. T., Harmon, J. K., Coman, E. I., Cahill, J. T. S., and Ernst, C. M. (2013). A comparison of rayed craters on the moon and mercury. J. Geophys. Res.: Planets, 118(10), 2247-2261. https://doi.org/10.1002/jgre.20166

Nozette, S., Spudis, P., Bussey, B., Jensen, R., Raney, K., Winters, H., Lichtenberg, C. L., Marinelli, W., Crusan, J., ... Robinson, M. (2010). The Lunar Reconnaissance Orbiter Miniature Radio Frequency (Mini-RF) technology demonstration. Space Sci. Rev., 150(1-4), 285-302. https://doi.org/10.1007/s11214-009-9607-5

Oberbeck, V. R. (1975). The role of ballistic erosion and sedimentation in lunar stratigraphy. Rev. Geophys., 13(2), 337-362. https://doi.org/10.1029/RG013i002p00337

Paige, D. A., Foote, M. C., Greenhagen, B. T., Schofield, J. T., Calcutt, S., Vasavada, A. R., Preston, D. J., Taylor, F. W., Allen, C. C., ... McCleese, D. J. (2010). The lunar reconnaissance orbiter diviner lunar radiometer experiment. Space Sci.
Rev., 150(1), 125-160. https://doi.org/10.1007/s11214-009-9529-2

Petro, N. E., and Pieters, C. M. (2006). Modeling the provenance of the Apollo 16 regolith. J. Geophys. Res.: Planets, 111(E9), 1-13. https://doi.org/10.1029/2005JE002559

Pike, R. J. (1974). Ejecta from large craters on the Moon: Comments on the geometric model of McGetchin et al. Earth Planet. Sci. Lett., 23(3), 265-271. https://doi.org/10.1016/0012-821X(74)90114-9

Robinson, M. S., Brylow, S. M., Tschimmel, M., Humm, D., Lawrence, S. J., Thomas, P. C., Denevi, B. W., Bowman-Cisneros, E., Zerr, J., ... Hiesinger, H. (2010). Lunar Reconnaissance Orbiter Camera (LROC) instrument overview. Space Sci. Rev., 150(1), 81-124. https://doi.org/10.1007/s11214-010-9634-2

Schultz, P. H., and Gault, D. E. (1985). Clustered impacts: Experiments and implications. J. Geophys. Res.: Solid Earth, 90(B5), 3701-3732. https://doi.org/10.1029/JB090iB05p03701

Spudis, P. D. (1994). The large impact process inferred from the geology of lunar multiring basins. In B. O. Dressler, et al. (Eds.), Large Meteorite Impacts and Planetary Evolution: Boulder (pp. 293). Boulder: Geological Society of America.

Spudis, P. D., Wilhelms, D. E., and Robinson, M. S. (2011). The Sculptured Hills of the Taurus Highlands: Implications for the relative age of Serenitatis, basin chronologies and the cratering history of the Moon. J. Geophys. Res., 116, EOOH03. https://doi.org/10.1029/2011JE003903

Wilhelms, D. E. (1987). The geologic history of the Moon. U. S. Geol. Surv. Prof. Pap., 1348, 1-302.

Xiao, Z. Y., and Werner, S. C. (2015). Size-frequency distribution of crater populations in equilibrium on the Moon. J. Geophys. Res.: Planets, 120(12), 2277-2292. https://doi.org/10.1002/2015JE004860

Xiao, Z. Y., Zeng, Z. X., Ding, N., and Molaro, J. (2013). Mass wasting features on the Moon-how active is the lunar surface?. Earth Planet. Sci. Lett., 376, 1-11. https://doi.org/10.1016/j.epsl.2013.06.015

Xiao, Z. Y. (2016). Size-frequency distribution of different secondary crater populations: 1. equilibrium caused by secondary impacts. J. Geophys. Res.: Planets, 121(12), 2404-2425. https://doi.org/10.1002/2016JE005139

Xie, M. G., and Zhu, M. H. (2016). Estimates of primary ejecta and local material for the Orientale basin: Implications for the formation and ballistic sedimentation of multi-ring basins. Earth Planet. Sci. Lett., 440, 71-80. https://doi.org/10.1016/j.epsl.2016.02.012

Zhao, H. X., and Magoulès, F. (2011). New parallel support vector regression for predicting building energy consumption. In 2011 IEEE Symposium on Computational Intelligence in Multicriteria Decision-Making (pp. 14-21). Paris: IEEE. https://doi.org/10.1109/SMDCM.2011.5949289 